\title{
Density profile, velocity anisotropy and line-of-sight external convergence of SLACS gravitational lenses
}

\author{
Antonio C. C. Guimarães and Laerte Sodré Jr. \\ Departamento de Astronomia, Universidade de São Paulo, Rua do Matão 1226, CEP 05508-090 São Paulo \\ - SP, Brazil; aguimaraes@astro.iag.usp.br
}

\begin{abstract}
Data from 58 strong-lensing events surveyed by the Sloan Lens ACS Survey are used to estimate the projected galaxy mass inside their Einstein radii by two independent methods: stellar dynamics and strong gravitational lensing. We perform a joint analysis of these two estimates inside models with up to three degrees of freedom with respect to the lens density profile, stellar velocity anisotropy, and line-of-sight (LOS) external convergence, which incorporates the effect of the large-scale structure on strong lensing. A Bayesian analysis is employed to estimate the model parameters, evaluate their significance and compare models. We find that the data favor Jaffe's light profile over Hernquist's, but that any particular choice between these two does not change the qualitative conclusions with respect to the features of the system that we investigate. The density profile is compatible with an isothermal, being sightly steeper and having an uncertainty in the logarithmic slope of the order of $5 \%$ in models that take into account a prior ignorance on anisotropy and external convergence. We identify a considerable degeneracy between the density profile slope and the anisotropy parameter, which largely increases the uncertainties in the estimates of these parameters, but we encounter no evidence in favor of an anisotropic velocity distribution on average for the whole sample. An LOS external convergence following a prior probability distribution given by cosmology has a small effect on the estimation of the lens density profile, but can increase the dispersion of its value by nearly $40 \%$.
\end{abstract}

Subject headings: dark matter - galaxies: elliptical and lenticular, cD - galaxies: fundamental parameters - galaxies: kinematics and dynamics - galaxies: structure - gravitational lensing: strong

\section{Introduction}

The observation of strong gravitational lensing events has allowed many studies about the mass, density profile, and structure of the galaxies that act as lenses, which could also have important implications for dark matter and cosmology studies - see Kochanek's contribution in Meylan et al. (2006) for a review in the field. Among strong lensing works, and of particular interest here, there are those of joint studies with stellar dynamics, which allow a determination of the density profile for individual lenses (Romanowsky \& Kochanek 1999;

Trott \& Webster 2002; Koopmans \& Treu 2003;

Treu \& Koopmans 2002a, b; Koopmans et al. 2003; Czoske et al. 2008) and for sets of lenses (Koopmans et al. 2006; Gavazzi et al. 2007; Barnabè et al. 2009). It is of fundamental interest to this line of research to control for possible systematic effects, such as the influence of the large-scale structure (LSS) along the line-of-sight (LOS) and the role of other properties of the lens galaxy.

Several works have considered LOS effects on strong lensing studies. Bar-Kana (1996) investigated theoretically the effect of the LSS on strong-lensing events, finding that it can be significant, for example leading to incorrect conclusions about the distribution of matter in the lens. Keeton et al. (1997) observed that external shear due to galaxies and clusters associated with the primary lens or along the LOS can be an important perturbation in individual lens models. Premadi \& Martel (2004) used ray 
tracing to investigate the effect of density inhomogeneities along the LOS of strong lenses and concluded that the effect of environment is negligible in general, but might be important in rare cases. Wambsganss et al. (2005) noted that secondary matter along the LOS of strong lenses is strongly dependent on source redshift, being rare for sources with $z<1$, but that can lead in some circumstances to an overestimate of $10 \%-15 \%$ of the primary lens mass if ignored. Photometric and spectroscopic observations (Tonrv \& Kochanek 2000; Momcheva et al. 2006; Fassnacht et al. 2006; Williams et al. 2006; Auger et al. 2007) discovered a significant LOS effect on some individual strong lens galaxies. Moustakas et al. (2007) observed that even in underdense local environments, the LOS contamination may give a considerable contribution to galaxy-scale strong lenses. Using ray-tracing thought the Millennium Simulation, Hilbert et al. (2007) determined that strong-lensing LOSs are biased toward higher than average mean densities, contributing a few percent to the total surface density, and Puchwein \& Hilbert (2009) found that secondary matter along the LOS has a large effect on the strong-lensing optical depth and the cross section for cluster strong lensing. Auger (2008) did not find an overdensity of photometric sources along the LOS of a limited sample of SLACS strong lenses in comparison with other Sloan digital Sky Survey (SDSS) massive early-type galaxies and interpreted that as evidence against a possible LOS contamination. Treu et al. (2009) measured the overdensity of galaxies around SLACS lenses and observed that typical contributions from external mass distributions are of the order of few percent, but reaching $10 \%-20 \%$ in some cases. Faure et al. (2009) considered strong- and weaklensing observations in the COSMOS survey and compared with simulations, finding that stronglensed images with large angular separation were in the densest regions.

In this paper, we use two independent galaxy mass estimate methods, strong gravitational lensing, and stellar dynamics to examine the influence of the LSS in the LOS of the lenses and its effect on the determination of the lens density profile. We use all the suitable events in the SLACS sample, considering realistic brightness functions for the lens galaxies, and incorporating our prior ig- norance on their stellar velocity anisotropy.

In Section 2 we describe how we calculate the mass of SLACS lenses using strong gravitational lensing and stellar dynamics, and our Bayesian statistical approach. In Section 3 we show our results, and in Section 4 we present our discussion and conclusions.

\section{Data and Methods}

The analysis in this paper is based on the comparison of galaxy masses calculated through two different methods: gravitational lensing and dynamical analysis. In Section 2.1, we present the data used in the analysis, collected from the SLACS survey.

In Sections 2.2 and 2.3, we discuss the lensing and dynamical mass determinations, respectively. We will assume simple models for the galaxy mass distribution (e.g., spherical symmetry, power-law density distribution) because they have few free parameters and allow to illustrate well the two methods. For a similar approach see Koopmans et al. (2006).

We want to examine whether the two mass estimates are indeed equivalent and/or if there is evidence of systematic differences between them. Section 2.4 presents a Bayesian framework to analyze this problem.

\subsection{Data}

The selected set of galaxies is part of the Sloan Lens ACS Survey, SLACS (Bolton et al. 2006), which is a Hubble Space Telescope (HST) Snapshot imaging survey for strong gravitational galactic lenses. The candidates for the HST imaging were selected spectroscopically from the SDSS database and are a sub-sample of the SDSS Luminous Red Galaxy (LRG) sample.

We use data compiled from Koopmans et al. (2006), Gavazzi et al. (2007) and Bolton et al. (2008a), constructing a sample of 58 strong gravitational lensing events where the lenses are isolated early-type galaxies $(\mathrm{E}+\mathrm{S} 0)$. Data from SLACS are especially suitable for joint strong lensing and dynamical analysis because they allow precise determination ( $5 \%$ error) of the Einstein radius for each lens galaxy in a relatively homogeneous sample of early-type galaxies. And, at 
the same time, SDSS has precise stellar velocity dispersion measurements $(6 \%$ average error) for the lenses, as well as redshifts for lenses and background sources.

For each lens system we are interested in the redshift of the background lensed source $z_{s}$, the redshift of the lens $z_{l}$, the average stellar velocity dispersion inside an aperture $\sigma_{a p}$, the effective angular radius $\theta_{\text {ef }}$ and the Einstein angular radius $\theta_{E}$. The sample average values for these quantities are $\left\langle z_{l}\right\rangle=0.2,\left\langle z_{s}\right\rangle=0.6,\left\langle\sigma_{a p}\right\rangle=250 \mathrm{kms}^{-1}$, $\left\langle\theta_{e f}\right\rangle=2.2^{\prime \prime}$, and $\left\langle\theta_{E}\right\rangle=1.2^{\prime \prime}$.

The source and lens redshifts were determined from the SDSS spectra, and the stellar velocity dispersion corresponds to the light-weighted average inside the $3^{\prime \prime}$ diameter SDSS fiber.

\subsection{Lensing Mass}

The estimated projected mass inside the Einstein radius $R_{E}=\theta_{E} D_{L}$, is given by

$$
M_{E}=\pi R_{E}^{2} \Sigma_{c r},
$$

where

$$
\Sigma_{c r}=\frac{c^{2}}{4 \pi G} \frac{D_{S}}{D_{L} D_{L S}},
$$

and $D_{[L, S, L S]}$ is the angular-diameter distance of the lens, source, and between lens and source, respectively. These distances are calculated assuming a redshift-distance relation derived inside a chosen cosmological model that in the present paper is a concordance $\Lambda \mathrm{CDM}$ model with $\Omega_{m}=0.3$, $\Omega_{\Lambda}=0.7$.

The Einstein radii were determined from $H S T$ images using strong lensing modeling of the lenses and reconstruction of the unlensed sources (Koopmans et al. 2006; Gavazzi et al. 2007). The uncertainties on $\theta_{E}$ were reported to be around $5 \%$, so we use this value for all Einstein radii when calculating the error on $M_{E}$. Note that the lensing modeling uses a Singular Isothermal Ellipsoid (SIE) mass model, but the resulting projected mass distribution is parameterized by an Einstein radius so that the enclosed mass in the projected ellipse is the same that would be enclosed in a projected circle from an equivalent Singular Isothermal Sphere. This is the radius we adopt here. Indeed, the Einstein radius determined this way is a robust attribute of the lens, being little sensitive to the lens model used (see Kochanek's contribution in Meylan et al. (2006)).

However the Einstein mass captures not only the lensing effect of the lens galaxy, but also the lensing effect of all LOSs over- and underdensities. We model this LSS contribution by subtracting an effective external lensing convergence given by cosmology, $\kappa_{\text {ext }}$. The real lensing mass of the lens galaxy is therefore given by (Suyu et al. 2010).

$m_{L}\left(\kappa_{\text {ext }}\right)=M_{E}-\pi R_{E}^{2} \kappa_{e x t} \Sigma_{c r}=\left(1-\kappa_{\text {ext }}\right) M_{E}$.

Note that the external convergence field can assume positive and negative values, therefore it can both decrease or increase the lensing mass. In general we do not know the value of $\kappa_{\text {ext }}$ for a given lens, so it has to be treated as a random value drawn from a probability distribution. That will be further discussed in Section 2.4.

\subsection{Dynamical Mass}

We call the dynamical mass, $m_{D}$, the mass estimated from the observed velocity dispersion. Here we are interested in examining the case of a power law for the density profile, $\rho=A r^{\gamma}$, where $A$ is a constant that has to be determined from the Jeans equation and the observed velocity dispersion. The mass within the cylinder $C_{E}$ of Einstein radius $R_{E}$ is then

$m_{D}(\gamma, \beta)=\int_{C_{E}} \rho(r) d V=\frac{2 \pi^{3 / 2}}{3+\gamma} \frac{\Gamma\left(-\frac{1+\gamma}{2}\right)}{\Gamma\left(-\frac{\gamma}{2}\right)} A R_{E}^{3+\gamma}$.

The spherical Jeans equation Binney \& Tremaine 1987) can be written as

$$
\frac{1}{\nu} \frac{d\left(\nu \sigma_{r}^{2}\right)}{d r}+2 \frac{\beta \sigma_{r}^{2}}{r}=-\frac{d \Phi}{d r}=-\frac{\pi G}{3+\gamma} A r^{1+\gamma},
$$

where $\sigma_{r}$ is the radial velocity dispersion, $\nu(r)$ is the luminosity density profile (Jaffe 1983; Hernquist 1990), $\beta \equiv 1-\sigma_{t}^{2} / \sigma_{r}^{2}$ is the anisotropy parameter of the velocity distribution ( $\sigma_{t}$ is the tangential velocity dispersion), and $\Phi$ is the gravitational potential produced by the assumed density profile.

Since the quantity observationally available is the luminosity-weighted average velocity dispersion within a given aperture, $\sigma_{a p}^{2}$, the following constraint is necessary for the determination of the 


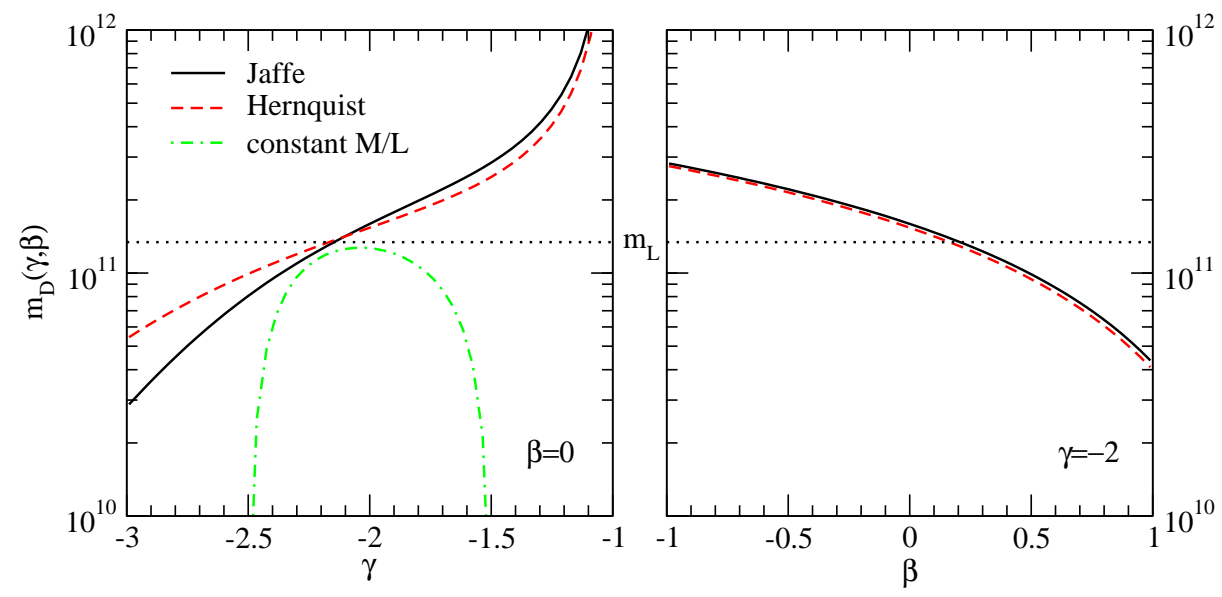

Fig. 1.- Dynamical mass behavior in relation to density profile logarithmic slope, $\gamma$, and the velocity anisotropy parameter, $\beta$, in units of $h^{-1} M_{\odot}$. The curves were calculated for an "average" system with $z_{l}=0.2, z_{s}=0.6, \sigma_{a p}=250 \mathrm{kms}^{-1}, \theta_{E}=1.2^{\prime \prime}, \theta_{e f}=2.2^{\prime \prime}$. Solid lines use Jaffe and dashed lines use Hernquist light distribution profiles. The dotted line depicts the strong lensing mass, Equation (10), for the "average" system: $M_{E}=1.34 \cdot 10^{11} h^{-1} M_{\odot}$. The dot-dashed line on the left panel was obtained using $\nu(r) \propto \rho(r) \propto r^{\gamma}$.

constant $A$ :

$$
\sigma_{a p}^{2}=\frac{\int_{C_{a p}} \nu \sigma_{r}^{2} d V}{\int_{C_{a p}} \nu d V},
$$

where the integration volume is an infinite cylinder of radius $R_{a p}$ with axis along the LOS.

To simplify, and since there is very little prior knowledge on the velocity anisotropy parameter, we assume that $\beta$ is a constant. In Appendix A.1. we give more details on the solution of Jean's Equation (5), and in Appendix A.2 we examine the correction due to seeing effects.

Figure 1 displays the general behavior of the dynamical mass as a function of the density profile slope and velocity anisotropy parameter. We examine $m_{D}(\gamma, \beta=0)$ and $m_{D}(\gamma=-2, \beta)$. Other combinations around these fixed values give qualitatively similar results. The dotted line depicts the strong-lensing mass for this hypothetical system, so it is possible to glimpse from the intersection of the curves the expected value of the dynamical parameters, $\gamma$ and $\beta$. The use of the Jaffe or the Hernquist light profiles do not change the qualitative behavior of the curves. The use of a constant mass to light ratio, $\nu(r) \propto \rho(r) \propto r^{\gamma}$, displayed as the dot-dashed line in the left panel of Figure 1, gives a very distinct and interesting behavior for $m_{D}(\gamma)$, reproducing a result obtained by Guimarães \& Sodré (2007). However, a single power law is not a realistic approximation for the light distribution of the lens galaxies in the sample.

\subsection{Statistical Analysis}

We want to compare the estimates of lensing and dynamical masses taking into account the possibility that masses obtained through gravitational lensing are affected by the LSS, $m_{L}=m_{L}\left(\kappa_{\text {ext }}\right)$, and that the lens galaxy dynamical mass depends only on its density profile and velocity anisotropy, $m_{D}=m_{D}(\gamma, \beta)$. Our models, then, can have up to three free parameters: $\kappa_{e x t}, \gamma$ and $\beta$.

To construct a likelihood for the system we define the quantity

$$
F=\frac{m_{L}}{m_{D}}-\frac{m_{D}}{m_{L}} .
$$

Note that both $m_{L}$ and $m_{D}$ refer to the projected mass within the Einstein radius. The likelihood 
for each lens system is then written as

$$
\mathcal{L}_{i}=\frac{1}{\sqrt{2 \pi} \sigma_{F, i}^{2}} \exp \left[-\frac{\left(F_{t}-F_{o b s, i}\right)^{2}}{2 \sigma_{F, i}^{2}}\right],
$$

where $F_{\text {obs }}=F_{\text {obs }}\left(\kappa_{\text {ext }}, \gamma, \beta ;\right.$ data $)$ is the measured $F$ given the model and the observational data, and $F_{t}$ is the expected value for it, which, in the desired case where both $m_{L}$ and $m_{D}$ are estimates of the same true galaxy mass, corresponds to $F_{t}=0$. Note that other quantities could be defined to construct the likelihood, for example $F=m_{L} / m_{D}+m_{D} / m_{L}\left(F_{t}=2\right), F=m_{L} / m_{D}$ $\left(F_{t}=1\right), F=m_{L}-m_{D}\left(F_{t}=0\right)$. The next to last definition gives a likelihood that is not symmetrical between $m_{L}$ and $m_{D}$, what is not desirable, and the last example has the inconvenience of maximizing the likelihood not only in the desired region of the parameter space in which $m_{L} \sim m_{D}$, but also in the region where both mass estimates are small, what introduces artificial solutions that give maximum LOS contamination. However if $\kappa_{\text {ext }}=0$ is fixed, then all definitions for $F$, including the last, give very similar results.

The variance in $F$ is estimated as being (an index $i$ is implied in all quantities)

$$
\sigma_{F}^{2}=\frac{\left(m_{L}^{2}+m_{D}^{2}\right)^{2}}{m_{L}^{4} m_{D}^{4}}\left(m_{L}^{2} \sigma_{D}^{2}+m_{D}^{2} \sigma_{L}^{2}\right),
$$

where $\sigma_{\{D, L\}}$ is the uncertainty in $m_{\{D, L\}}$.

The joint likelihood for the whole set of $N$ galaxies is then

$$
\mathcal{L}\left(\kappa_{\text {ext }}, \gamma, \beta\right)=\prod_{i=1}^{N} \mathcal{L}_{i} .
$$

Figure 2 shows the individual $\gamma$ likelihood distributions for each lens, as well as the joint likelihood for the whole sample, imposing fixed null external convergence and velocity anisotropy, recreating a similar figure obtained by Koopmans et al. (2009). In the same figure we examine the impact of the seeing correction (see Appendix A.2). The two narrow and almost indistinguishable curves differ just in that in their calculation one takes into account the seeing correction (dashed line) and the other (solid line) does not. The seeing correction is negligible.

The posterior probability distribution of the model parameters given the data is determined by

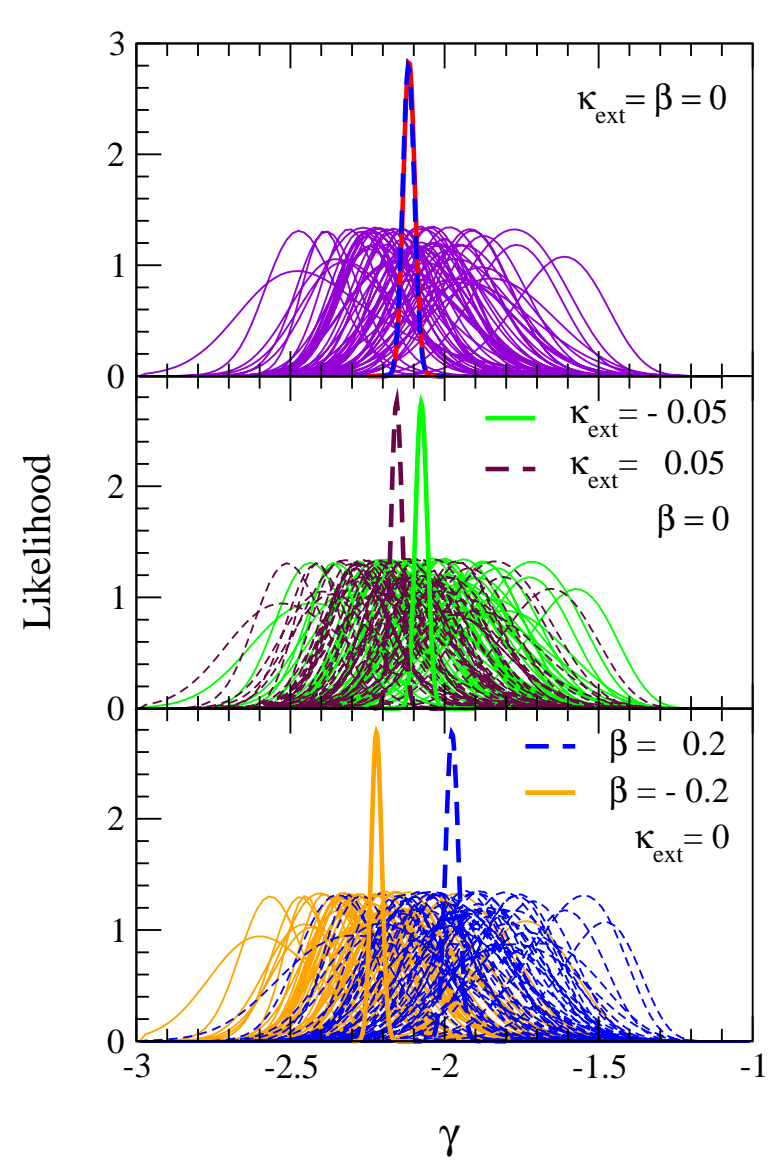

Fig. 2.- Likelihood distribution of $\gamma$ values. Multiple thin lines are the individual likelihoods for each lens. The thick spiky curves are the likelihoods for the whole set (rescaled for better visualization). It was adopted Jaffe's light profile in all panels. Each panel depicts particular cuts in the parameter space, indicated in the internal legend. In the top panel, the solid line does not take seeing correction into account and the dashed line does. 
the Bayes' theorem

$$
P\left(\kappa_{e x t}, \gamma, \beta\right)=\frac{\pi\left(\kappa_{e x t}, \gamma, \beta\right) \mathcal{L}\left(\kappa_{e x t}, \gamma, \beta\right)}{E},
$$

where

$$
E \equiv \int \pi\left(\kappa_{\text {ext }}, \gamma, \beta\right) \mathcal{L}\left(\kappa_{\text {ext }}, \gamma, \beta\right) d \kappa_{\text {ext }} d \gamma d \beta
$$

is the Bayesian evidence (Trotta 2008) and $\pi\left(\kappa_{e x t}, \gamma, \beta\right)=\pi\left(\kappa_{e x t}\right) \pi(\gamma) \pi(\beta)$ is the product of the prior probability distributions for the model parameters.

We adopt uniform priors for two of the parameters, $\pi(\gamma)=\pi(\beta)=1 / 2$ for $\gamma \in[-3,-1]$ and for $\beta \in[-1,1]$ and $\pi(\gamma)=\pi(\beta)=0$ outside these intervals. For $\kappa_{\text {ext }}$ we assume a prior given by the distribution of expected values for the convergence on a random LOS in the concordance cosmology adopted here (null mean value). We approximate this external convergence probability distribution function by the lognormal expression given by Taruya et al. (2002), $\pi\left(\kappa_{\text {ext }}\right)=P_{l n}\left(\kappa_{\text {ext }}\right)$, with $\left\langle\kappa^{2}\right\rangle^{1 / 2}=0.025$ and $\kappa_{\min }=-0.06$.

We estimate the free parameters $p$ of the models using the Bayes estimator (also called the posterior mean)

$$
\langle p\rangle \equiv \int P(p) p d p,
$$

and also calculate the root mean square deviation, $\operatorname{rms}(p)=\sqrt{\left\langle p^{2}\right\rangle-\langle p\rangle^{2}}$, which give a measure of the parameter value dispersion. Note that the parameter estimate is made from the whole data set considered altogether and not for each individual galaxy-lens system. The models are meant to be underlying models for all systems, independently of the particularities of each lens. In this approach the information about the sample dispersion among all lenses is mixed with the information about individual uncertainty in each lens.

Two models, $M_{1}$ and $M_{2}$, even with different degrees of freedom, can be compared by the ratio of their Bayesian evidence, also known as Bayes factor,

$$
B\left(M_{1}, M_{2}\right)=\frac{E\left(M_{1}\right)}{E\left(M_{2}\right)},
$$

in which values can be interpreted qualitatively using Jeffrey's scale (Trotta 2008). The strength of the evidence in favor of the model with larger $E$ is called inconclusive if $|\ln B|<1$, weak if above this value and below 2.5 , moderate if $2.5<|\ln B|<5$, and strong if above that.

The main appeal of this approach for model comparison is that the Bayesian evidence automatically implements Occam's razor by penalizing more strongly more complex models, those with more free parameters.

We sample the parameter space using a grid finer than the typical features scales of the system, which are characterized by the rms of each parameter. For example, for models with three free parameters we use as grid spacing: $\delta \kappa_{\text {ext }}=0.0036$, $\delta \gamma=0.018, \delta \beta=0.03$; for a model with only $\gamma$ free we use $\delta \gamma=0.004$. Such grids allow a complete sampling of the relevant parameter space and guarantees an appropriate probing of the likelihood and reliable estimates of parameter values and Bayesian evidence.

\section{Results}

We have analyzed the data described in Section 2.1 with models with up to three degrees of freedom, one $\left(\kappa_{\text {ext }}\right)$, corresponding to the LOS-LSS contamination, that would affect the strong lensing estimate of the lens mass and two $(\gamma$ and $\beta)$, corresponding to intrinsic properties of the lens, that determine its dynamical mass estimate. Two light distribution profiles (Jaffe and Hernquist) were examined.

Table1 summarizes some results for the models considered. The simplest model with no free parameters (isothermal density profile, no external convergence and velocity anisotropy) has a much lower Bayesian evidence than more complex models, so it can be said that it is strongly disfavored. A model with free density profile logarithmic slope has a much higher Bayesian evidence and yields a determination of this parameter with a $1 \%$ precision, being slightly steeper than an isothermal profile. This result is similar to what was found by Koopmans et al. (2009).

If instead of estimating $\langle\gamma\rangle$ and $\operatorname{rms}(\gamma)$ from Equation (13) we find for each lens galaxy a $\gamma_{i}$ (the point of maximum likelihood for each $\mathcal{L}_{i}$ ) and then proceed the calculation of the average value and sample deviation, we find -2.12 and 0.17 , respectively. That is in broad agreement with what is found from the posterior probability for the whole data set (11) (remember that there is a factor $\sqrt{N}$ 
Table 1: Models' Results Summary. Parameters posterior mean and rms, and Bayesian evidence. Values inside parentheses indicate fixed values in the model.

\begin{tabular}{lclllllll}
\hline \hline Free Parameters & Light Profile & $\left\langle\kappa_{\text {ext }}\right\rangle$ & $\operatorname{rms}\left(\kappa_{\text {ext }}\right)$ & $\langle\gamma\rangle$ & $\operatorname{rms}(\gamma)$ & $\langle\beta\rangle$ & $\operatorname{rms}(\beta)$ & $\ln E$ \\
\hline 0 & Jaffe & $(0)$ & $\ldots$ & $(-2)$ & $\ldots$ & $(0)$ & $\ldots$ & -49.7 \\
1 & Jaffe & $(0)$ & $\ldots$ & -2.115 & 0.019 & $(0)$ & $\ldots$ & -36.1 \\
2 & Jaffe & -0.005 & 0.022 & -2.111 & 0.026 & $(0)$ & $\ldots$ & -36.1 \\
2 & Jaffe & $(0)$ & $\ldots$ & -2.06 & 0.12 & 0.07 & 0.19 & -37.3 \\
3 & Jaffe & -0.003 & 0.023 & -2.06 & 0.12 & 0.06 & 0.19 & -37.4 \\
3 & Hernquist & -0.004 & 0.022 & -2.16 & 0.11 & -0.06 & 0.14 & -39.8 \\
\hline
\end{tabular}

between the sample and standard deviations before comparing with the value in Table 11). The sample deviation found can be considered a reasonable estimate of the sample dispersion on $\gamma \mathbf{1}$

The addition of a degree of freedom due to an external convergence, constrained by a prior probability, generating a model with two degrees of freedom, does not alter the mean density profile slope, but increases in almost $40 \%$ the uncertainty in its determination.

A model with no external convergence and freedom in the density profile and anisotropy parameter allows a profile closer to isothermal, and in fact compatible with it, given its much larger dispersion on $\gamma$ (six times larger than for a model with only $\gamma$ free). Such model is compatible with isotropy, but is weakly disfavored in relation to a model with free density profile and fixed null anisotropy. We note that Koopmans et al. (2009) found a positive anisotropy (significantly distinct from isotropy) for the same sample, but using for that an independent determination based on scaling relations of the density profile logarithmic slope. That is a different method from ours, which relies solely on the joint strong lensing and dynamical analysis.

A more complex model, with three degrees of freedom, does not give results much different from those of the model with free $\gamma$ and $\beta$ and fixed $\kappa_{e x t}=0$. A similar model, but with a different luminosity density profile (Hernquist), has a slightly steeper density profile and more negative anisotropy parameter, being weakly disfavored in comparison to the model with Jaffe's light profile.

\footnotetext{
${ }^{1}$ Note that Equation (7) of Koopmans et al. (2006) to obtain the intrinsic scatter leads approximately to the sample deviation if $\delta \gamma_{i} \ll \sigma_{\gamma}$.
}

If both external convergence and anisotropy are fixed, $\kappa_{\text {ext }}=\beta=0$, then $\langle\gamma\rangle=-2.12$ for both light profiles and $\operatorname{rms}(\gamma)=0.019$ (using Jaffe) and $\operatorname{rms}(\gamma)=0.027$ (using Hernquist). Therefore, the neglect of our ignorance on $\beta$ and, to a lower extent, $\kappa_{\text {ext }}$, by fixing them equal to zero, implies a considerable underestimate of the dispersion in $\gamma$ and possibly the introduction of a systematical error, since in the more complex model $\gamma=-2.06 \pm 0.12$ (using Jaffe) and $\gamma=-2.16 \pm 0.11$ (using Hernquist).

The results for the posterior mean and rms for the external convergence suggest that the system likelihood did not alter the prior probability distribution for $\kappa_{\text {ext }}$, which had $\left\langle\kappa_{\text {ext }}\right\rangle=0$ and $\left\langle\kappa_{\text {ext }}^{2}\right\rangle^{1 / 2}=0.025$. The small difference may be attributed to the computational truncation of the calculations at $\kappa_{\text {ext }} \sim 0.15$, excluding rare events of larger value.

Figure 3 shows posterior probability contours in two dimensions (free parameters in a model) for models using Jaffe's light profile (the use of Hernquist's would not change the conclusions). The top row panels have models with two free parameters and one fixed, and the bottom panels have the models with three free parameters, but one of them marginalized over. When we compare the top and bottom panels at the first column it shows that the prior ignorance on the anisotropy parameter implies a more uncertain determination of the density profile. The second column shows that the reciprocal is also true, and the third column clearly depicts this degeneracy between density profile and anisotropy parameter, which was already hinted in Figure 1, since the curves for $m_{D}(\gamma, \beta=0)$ and $m_{D}(\gamma=-2, \beta)$ have monotonically increasing and decreasing behavior, respectively. The effect of taking into account a prior 

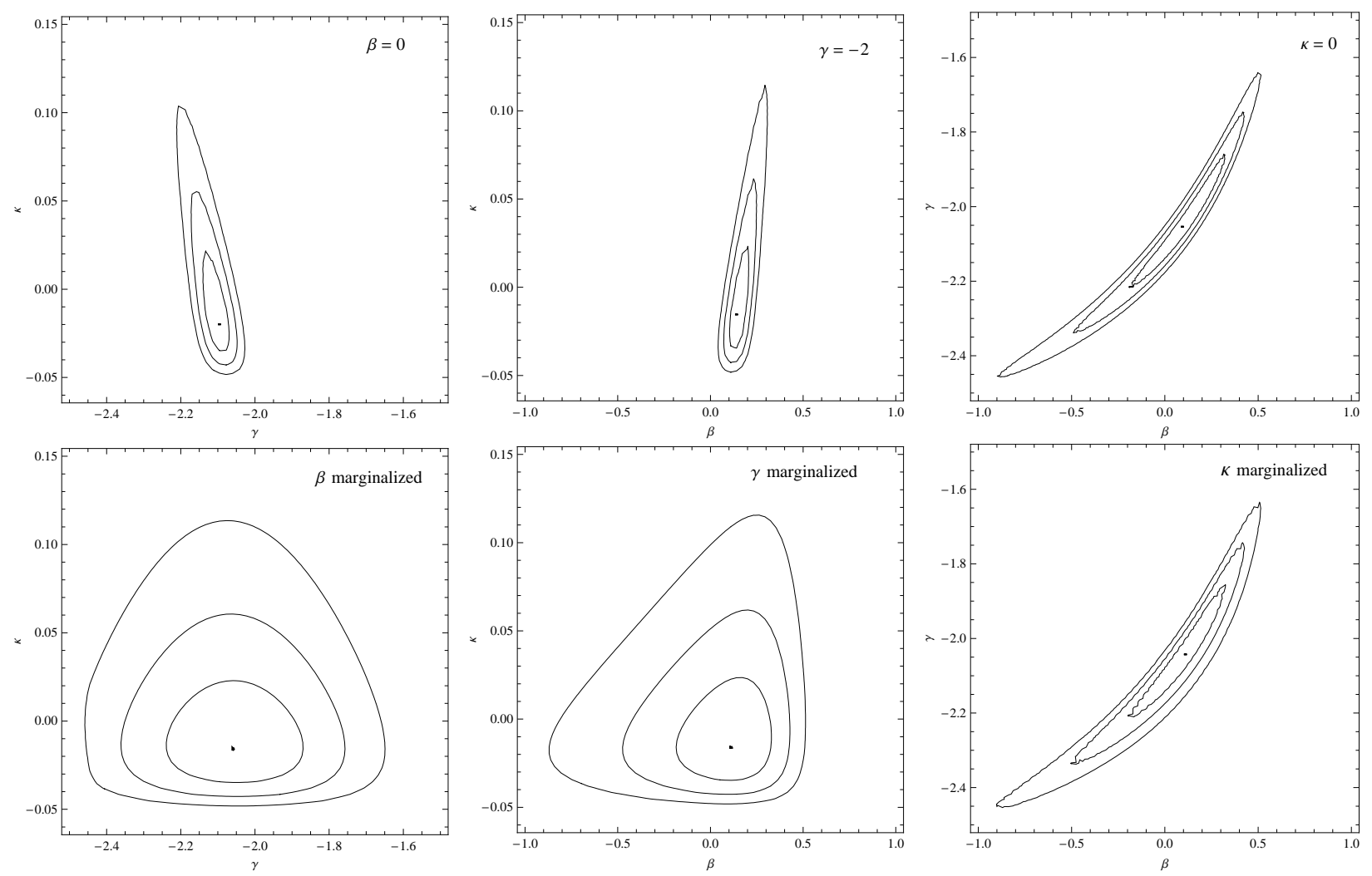

Fig. 3.- Posterior probability distribution. Top row has one of the three free parameters fixed. Bottom row has one of the three free parameters marginalized over. The point of maximum probability and the $1 \sigma$, $2 \sigma$ and $3 \sigma$ confidence levels are shown. Jaffe's light distribution profile is used.
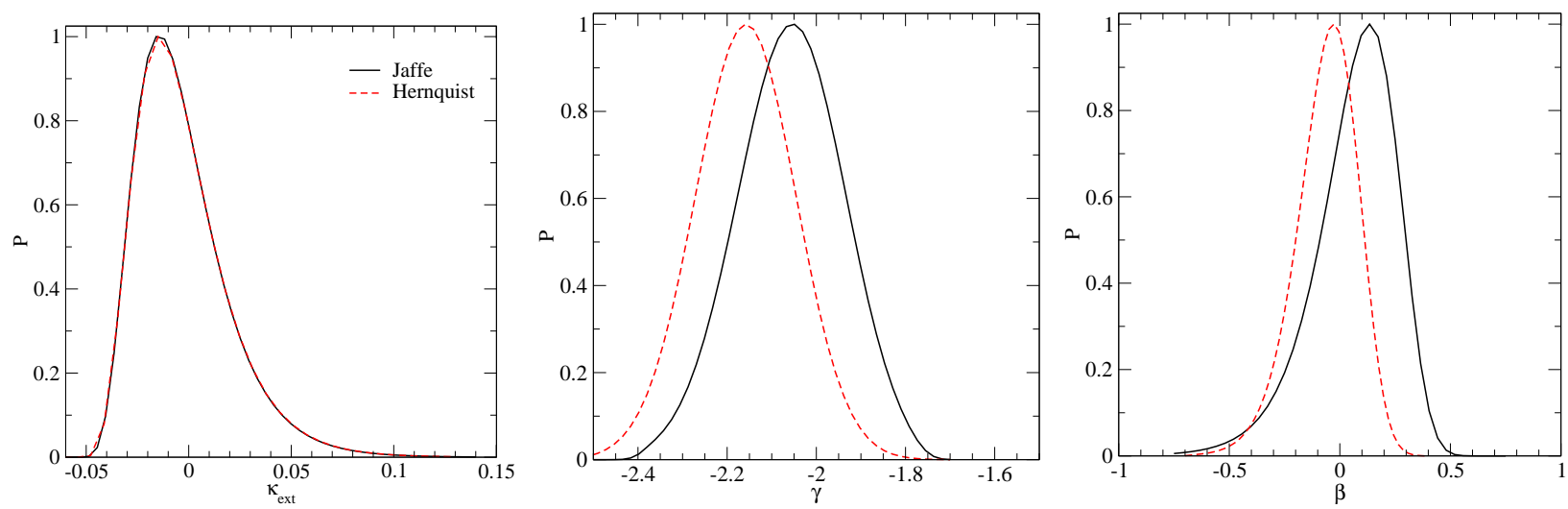

Fig. 4.- Posterior probability distribution marginalized over two parameters. Solid lines use Jaffe's light profile and dashed lines use Hernquist's. 
ignorance on $\kappa_{\text {ext }}$ is to fatten the banana-shaped contour of the $\gamma \times \beta$ posterior probability plot, but not changing the projected limits on $\gamma$ and $\beta$.

In Figure 4 are shown the parameter posterior probability distributions marginalized over two parameters for models with three degrees of freedom. Results for both light profiles (Jaffe and Hernquist) are presented, being qualitatively the same. The posterior distribution for the external convergence along the lenses LOSs (left panel) is indistinguishable from the prior distribution used, which indicates that the likelihood distribution in $\kappa_{\text {ext }}$ is much broader than the prior used.

The marginalized posterior probability distributions for the density profile logarithmic slope (central panel of Figure 4) follow a near Gaussian behavior and illustrates what was already observed in Table 1. The use of Hernquist's light profile implies a slightly steeper lens density profile than Jaffe's profile.

The right panel of Figure 4 shows the marginalized posterior probability distributions for the anisotropy parameter. It shows that our prior ignorance on the velocity anisotropy, quantified as a flat prior in the interval $[-1,1]$, is somewhat reduced, that the lenses are on average compatible with isotropy, but that positive and negative anisotropies are allowed, and that the use of Jaffe's light profile favors slightly more positive values for $\beta$ than Hernquist's.

\section{Discussion and Conclusions}

Two independent methods, strong gravitational lensing and stellar dynamics, were used to determine the projected galaxy mass within its Einstein radius for a set of 58 galaxies from SLACS. From the comparison of the two masses, the lens density profile, velocity anisotropy and LOS external convergence were probed in an integrated Bayesian approach.

The use of a prior probability distribution for the external convergence allowed us to quantify the effect of the LSS on the problem of joint lensing and dynamical analysis of SLACS lenses. The data do not seem have enough information to increase our knowledge on the external convergence for the particular set of LOS probed by the lenses since the posterior probability did not differ from the prior. The shapes of the $\kappa_{e x t} \times \gamma$ and $\kappa_{e x t} \times \beta$ degeneracies indicate that the determination of the density profile and anisotropy parameter are not very sensitive to the value of the LOS convergence in the range allowed by the prior probability distribution used. This can be understood from the functional dependence of the dynamical mass with $\gamma$ and $\beta$ (Figure 1). A small variation in $\gamma$ or $\beta$ implies a large change in $m_{D}$. This may mean that the uncertainties in the system due to the lens structure are more determinant than the role played by the external convergence and/or that SLACS lenses are in fact an unbiased sample in relation to a random LOS, despite the fact that the lenses are elliptical galaxies which are often found in dense regions. That would be in agreement with Treu et al. (2009) who find large external convergence values in just few extreme cases and with Wambsganss et al. (2005), for whom secondary matter along the LOS of strong lenses is strongly dependent on source redshift, being rare for sources with $z<1$, which is the case for most SLACS lenses. Another argument for why SLACS would not be biased to be in LOS with higher than average densities, as would be suggested by some theoretical works (Hilbert et al. 2007), is that they were spectroscopically selected. In contrast, lenses selected by photometric image identification would be more prone to be biased toward higher than average overdense LOS because lensed images of larger separation angle, tracing the densest regions (Faure et al. 2009), would be more easily identified, which does not happen in a spectroscopically selected sample.

The joint strong lensing and stellar dynamics analysis does improve our prior knowledge on the anisotropy parameter, but does not strongly constrain it, its probability distribution being broad and statistically compatible with isotropy on average. Nevertheless, the degeneracy between $\gamma$ and $\beta$ gives an indeterminacy that correlates a larger anisotropy with a flatter density profile, which can also be understood from the functional behavior of $m_{D}(\gamma, \beta)$ (Figure 1). An increment in $\gamma$ can be annulled, in terms of a variation in the dynamical mass, by a decrement in $\beta$, and vice versa.

The inclusion of two degrees of freedom in the model with respect to the LSS convergence and velocity anisotropy allows us to take into account and examine the effect of our prior ignorance on these features of the lens system. The 
most visible effect of $\kappa_{e x t}$ and $\beta$ on the determination of the density profile logarithmic slope is the considerable broadening of its probability distribution, which means a more uncertain determination of $\gamma$ than what is suggested by a simpler model that does not take into account those features. Within the uncertainty found in the more complex model, the density profile is statistically compatible with an isothermal profile. This very particular density profile is also found by other authors, apparently as a result of the complementarity of baryonic and dark matter profiles (Hamana et al. 2005; Ferreras et al. 2005; Lintott et al. 2006; Baltz et al. 2009; Czoske et al. 2008).

We used standard assumptions and approximations in our modeling of galaxies and analysis. Nevertheless, we can identify several areas where further work can be done to refine the understanding of these strong-lensing systems, which can also be seen as caveats to the present works in the area. Among them we highlight (1) the triaxiality and substructure of lens halos, whose importance was already suggested by Meneghetti et al. (2005) and Yencho et al. (2006) in the context of simulations on cluster scales, (2) the correction of the dynamical mass estimate due to rotational support. It is well known that some early-type galaxies can have a significant rotational component (e.g., Emsellem et al. 2007) and two-dimensional kinematics for some few SLACS lenses are already becoming available (Czoske et al. 2008; Barnabè et al. 2009), (3) the brightness distribution, which could be treated more realistically with the observed full surface luminosities for the individual lenses, instead of individualized fits of a universal profile, and (4) the velocity anisotropy parameter, which was assumed to be a constant, but that more realistically must be a function of radius. However, very little is known about the velocity anisotropy of earlytype galaxies, be it observationally, theoretically, and even from simulations.

The relaxation of some of our assumptions, with the almost inevitable addition of extra free parameters, could prove a fruitful source of investigation; however, it would likely require a larger galaxy sample to reduce the likely increased degeneracies among the degrees of freedom of the model. As we have illustrated, models with a large number of parameters may have a higher likelihood but lower Bayesian evidence, since the added complexity must pay its price in a Bayesian sense.

The authors thank CNPq and FAPESP for financial support, the SLACS and SDSS teams for the databases used in this work, and the referee whose comments helped to clarify many aspects of this paper. 


\section{Appendix}

\section{A.1. Solving Jean's Equation}

The spherical Jeans Equation (5) can be rewritten, defining $x \equiv r / R_{e f}$ (dimensionless) and

$$
y \equiv \frac{3+\gamma}{4 \pi G A} R_{e f}^{-(2+\gamma)}\left(\nu \sigma_{r}^{2}\right),
$$

as

$$
\frac{d y}{d x}=-2 \beta \frac{y}{x}-\nu x^{1+\gamma} .
$$

The luminosity distribution is well approximated by the profiles (Koopmans et al. 2006)

$$
\nu(x) \propto \frac{1}{x^{\gamma_{*}}\left(x+x_{*}\right)^{4-\gamma_{*}}},
$$

where $\gamma_{*}=1$ and $x_{*}=1 / 1.8153$ (Hernquist 1990), or $\gamma_{*}=2$ and $x_{*}=1 / 0.7447$ (Jaffe 1983). We examine both profiles.

The first-order linear differential Equation (A2) has a solution

$$
y(x)=x^{-2 \beta}\left[C-\int \nu(x) x^{1+\gamma+2 \beta} d x\right],
$$

where $C$ is an arbitrary constant and the most evident boundary condition is $y(x \rightarrow \infty)=0$. The analytical solution for the integral in Equation (A4) with Equation (A3) for $\nu(r)$ has the hypergeometric function ${ }_{2} F_{1}$, which has a computationally demanding solution. Therefore, we solve Equation (A2) using a fourth-order Runge-Kutha algorithm, starting at $y(x=1000)=0$ and evolving $y(x)$ down to $x=0.01$.

The dynamical mass (Equation (40)) within the Einstein radius $R_{E}$ is then

$m_{D}(\gamma, \beta)=\frac{\pi^{1 / 2}}{2 G} \frac{\Gamma\left(-\frac{1+\gamma}{2}\right)}{\Gamma\left(-\frac{\gamma}{2}\right)} \sigma_{a p}^{2} R_{E}\left(\frac{R_{E}}{R_{e f}}\right)^{2+\gamma} \frac{\int_{C_{E}} \nu d V}{\int_{C_{E}} y d V}$,

and its error is estimated from the observational errors on $\sigma_{a p}$ and $R_{E}$ through error propagation.

\section{A.2. Seeing}

We model the effect of the seeing through a Gaussian smoothing of the galaxy projected luminosity. Therefore, the observed surface brightness profile is related to an intrinsic (no seeing) profile by

$$
I_{o b s}(\theta)=\frac{e^{-\theta^{2} / 2 \sigma_{s}^{2}}}{\sigma_{s}^{2}} \int_{0}^{\infty} I\left(\theta^{\prime}\right) \mathrm{I}_{0}\left(\frac{\theta \theta^{\prime}}{\sigma_{s}^{2}}\right) e^{-\theta^{\prime 2} / 2 \sigma_{s}^{2}} \theta^{\prime} d \theta^{\prime},
$$

where $I_{0}$ is the modified Bessel function of first kind, and $\sigma_{s}^{2}$ is the Gaussian seeing variance. We use $\sigma_{s}=0.64^{\prime \prime}$, which corresponds to an FWHM of $1.5^{\prime \prime}$.

The seeing correction of the average velocity dispersion within the observational aperture is then given by

$\frac{\sigma_{a p}^{2}}{\left(\sigma_{a p}^{2}\right)_{o b s}}=\frac{\int_{0}^{R_{a p}} \sigma_{p}^{2}(R) I(R) R d R}{\int_{0}^{R_{a p}}\left[\sigma_{p}^{2}(R) I(R)\right]_{o b s} R d R} \frac{\int_{0}^{R_{a p}} I_{o b s}(R) R d R}{\int_{0}^{R_{a p}} I(R) R d R}$,

where $\sigma_{p}^{2}$ is the projected velocity dispersion profile, $\left[\sigma_{p}^{2}(R) I(R)\right]_{o b s}$ is defined in an analogous way to $I_{o b s}(R)$ in Equation (A6), and the projection is calculated through (Binney \& Tremaine 1987)

$$
I(R) \sigma_{p}^{2}(R)=2 \int_{R}^{\infty}\left(1-\beta \frac{R^{2}}{r^{2}}\right) \frac{\nu \sigma_{r}^{2} r}{\sqrt{r^{2}-R^{2}}} d r .
$$

\section{REFERENCES}

Auger, M. W. 2008, MNRAS, 383, L40

Auger, M. W., Fassnacht, C. D., Abrahamse, A. L., Lubin, L. M., \& Squires, G. K. 2007, AJ, 134, 668

Baltz, E. A., Marshall, P., \& Oguri, M. 2009, JCAP, 1, 15

Bar-Kana, R. 1996, ApJ, 468, 17

Barnabè, M., Czoske, O., Koopmans, L. V. E., Treu, T., Bolton, A. S., \& Gavazzi, R. 2009, MNRAS, 399, 21

Binney, J. \& Tremaine, S. 1987, Galactic Dynamics (Princeton: Princeton Univ. Press)

Bolton, A. S., Burles, S., Koopmans, L. V. E., Treu, T., \& Moustakas, L. A. 2006, ApJ, 638, 703

Bolton, A. S., Burles, S., Treu, T., Koopmans, L. V. E., \& Moustakas, L. A. 2007, ApJ, 665, L105

Bolton, A. S., Burles, S., Koopmans, L. V. E., Treu, T., Gavazzi, R., Moustakas, L. A., Wayth, R., \& Schlegel, D. J. 2008, ApJ, 682, 964 
Bolton, A. S., Treu, T., Koopmans, L. V. E., Gavazzi, R., Moustakas, L. A., Burles, S., Schlegel, D. J., \& Wayth, R. 2008, ApJ, 684, 248

Czoske, O., Barnabè, M., Koopmans, L. V. E., Treu, T., \& Bolton, A. S. 2008, MNRAS, 384, 987

Emsellem, E., et al. 2007, MNRAS, 379, 401

Fassnacht, C. D., Gal, R. R., Lubin, L. M., McKean, J. P., Squires, G. K., \& Readhead, A. C. S. 2006, ApJ, 642, 30

Faure, C., et al. 2009, ApJ, 695, 1233

Ferreras, I., Saha, P., \& Williams, L. L. R. 2005, ApJ, 623, L5

Gavazzi, R., Treu, T., Rhodes, J. D., Koopmans, L. V. E., Bolton, A. S., Burles, S., Massey, R. J., \& Moustakas, L. A. 2007, ApJ, 667, 176

Guimarães, A. C. C., \& Sodré, L. J. 2007, arXiv:0706.3098

Hamana, T., Ohyama, Y., Chiba, M., \& Kashikawa, N. 2005, ArXiv Astrophysics eprints, arXiv:astro-ph/0507056

Hernquist, L. 1990, ApJ, 356, 359

Hilbert, S., White, S. D. M., Hartlap, J., \& Schneider, P. 2007, MNRAS, 382, 121

Jaffe, W. 1983, MNRAS, 202, 995

Keeton, C. R., Kochanek, C. S., \& Seljak, U. 1997, ApJ, 482, 604

Koopmans, L. V. E., \& Treu, T. 2003, ApJ, 583, 606

Koopmans, L. V. E., Treu, T., Fassnacht, C. D., Blandford, R. D., \& Surpi, G. 2003, ApJ, 599, 70

Koopmans, L. V. E., Treu, T., Bolton, A. S., Burles, S., \& Moustakas, L. A. 2006, ApJ, 649, 599

Koopmans, L. V. E., et al. 2009, ApJ, 703, L51

Lintott, C. J., Ferreras, I., \& Lahav, O. 2006, ApJ, 648,826
Meneghetti, M., Bartelmann, M., Jenkins, A., \& Frenk, C. 2007, MNRAS, 381, 171

Meylan, G., Jetzer, P., North, P., Schneider, P., Kochanek, C. S., \& Wambsganss, J. 2006, SaasFee Advanced Course 33: Gravitational Lensing: Strong, Weak and Micro

Momcheva, I., Williams, K., Keeton, C., \& Zabludoff, A. 2006, ApJ, 641, 169

Moustakas, L. A., et al. 2007, ApJ, 660, L31

Premadi, P., \& Martel, H. 2004, ApJ, 611, 1

Puchwein, E., \& Hilbert, S. 2009, MNRAS, 398, 1298

Romanowsky, A. J., \& Kochanek, C. S. 1999, ApJ, 516,18

Suyu, S. H., Marshall, P. J., Auger, M. W., Hilbert, S., Blandford, R. D., Koopmans, L. V. E., Fassnacht, C. D., \& Treu, T. 2010, ApJ, 711, 201

Taruya, A., Takada, M., Hamana, T., Kayo, I., \& Futamase, T. 2002, ApJ, 571, 638

Tonry, J. L., \& Kochanek, C. S. 2000, AJ, 119, 1078

Treu, T., \& Koopmans, L. V. E. 2002, MNRAS, 337, L6

Treu, T., \& Koopmans, L. V. E. 2002, ApJ, 575, 87

Treu, T., Gavazzi, R., Gorecki, A., Marshall, P. J., Koopmans, L. V. E., Bolton, A. S., Moustakas, L. A., \& Burles, S. 2009, ApJ, 690, 670

Trott, C. M., \& Webster, R. L. 2002, MNRAS, 334,621

Trotta, R. 2008, Contemporary Physics, 49, 71

Wambsganss, J., Bode, P., \& Ostriker, J. P. 2005, ApJ, 635, L1

Williams, K. A., Momcheva, I., Keeton, C. R., Zabludoff, A. I., \& Lehár, J. 2006, ApJ, 646, 85

Yencho, B. M., Johnston, K. V., Bullock, J. S., \& Rhode, K. L. 2006, ApJ, 643, 154

This 2-column preprint was prepared with the AAS LATEX macros v 5.2 . 